\title{
Peptoid-based hierarchically-structured biomimetic nanomaterials: Synthesis, characterization and applications
}

\author{
Jianli Liu ${ }^{1,2}$, Bin $\mathrm{Cai}^{2}$, Lifeng $\mathrm{Cui}^{{ }^{*}}$ and Chun-Long $\mathrm{Chen}^{2^{*}}$
}

ABSTRACT Peptoids (or poly- $\mathrm{N}$-substituted glycines) are a promising class of bioinspired sequence-defined polymers due to their highly efficient synthesis, high chemical stability, enzyme hydrolysis resistance, and biocompatibility. By tuning the side chain chemistry of peptoids, it allows for precise control over sequences and achieving a large side-chain diversity. Due to these unique features, in the last several years, many amphiphilic peptoids were designed as highly tunable building blocks for the preparation of biomimetic nanomaterials with well-defined hierarchical structures and desired functionalities. Herein, we provide an overview of the recent achievements in this area by dividing them into the following three aspects. First, mica- and silica-templated peptoid selfassembly are summarized. The presence of inorganic substrates provides the guarantee of investigating their selfassembly mechanisms and interactions between peptoids and substrates using nanoscale characterization techniques, particularly in situ atomic force microscopy (AFM) and AFMbased dynamic force spectroscopy (AFM-DFS). Second, solution-phase self-assembly of peptoids into nanotubes and nanosheets is presented, as well as their self-repair properties. Third, the applications of peptoid-based nanomaterials are outlined, including the construction of catalytic nanomaterials as a template and cytosolic delivery as cargoes.

Keywords: peptoid, self-assembly, biomimetic nanomaterial, nanoscale characterization, assembly mechanism

\section{INTRODUCTION}

Bioinspired materials assembled from sequence-defined polymers are attracting increasing attention due to significant advantages over their natural counterparts: the ability to precisely tune their structures over a broad range of chemical and physical properties, increased sta- bility, and improved processability [1-6]. Peptoids are non-natural mimetics of peptides and proteins composed of repeating $\mathrm{N}$-substituted glycine monomer units, where the side chain is covalently attached to the nitrogen atom rather than the $\alpha$-carbon (Fig. 1a) $[1,2,7]$. This subtle structural modification provides peptoids with unique structures and properties in contrast to peptides and proteins, such as high stability against protease digestion, enhanced chemical and thermal stability, and the lack of backbone hydrogen bond donors which simplifies the building of peptoid-peptoid and peptoid-surface interactions exclusively through side-chain chemistry. Because of such advantages, in the last several years, amphiphilic peptoids have been frequently designed and used as sequence-defined building blocks to assemble into biomimetic nanomaterials with various hierarchical structures both on the substrate surfaces and in the solution [1,2,718]. To access sequence-defined peptoids, a step-wise "sub-monomer" synthesis approach was developed by Zuckermann et al. [19] in 1992. This approach uses a two-step sub-monomer addition reaction to build peptoid side chains from primary amines, instead of amino acid precursors used in the solid-phase peptide synthesis (Fig. 1b). Because several hundreds of commercially available amines can be used for peptoid synthesis, this method provides the access to a nearly infinite diversity of peptoid sequences with precisely controlled side-chain chemistries, allowing a fine tuning of the structure and function of peptoid-based nanomaterials by incorporating protein-like high information content into the materials design $[3,20,21]$.

The recent development of peptoid-based nanomaterials was involved with many state-of-the-art nanoscale characterization techniques. Although traditional meth-

\footnotetext{
${ }^{1}$ School of Materials Science and Engineering, Dongguan University of Technology, Dongguan 523808, China

${ }^{2}$ Physical Sciences Division, Physical and Computational Sciences Directorate, Pacific Northwest National Laboratory, Richland WA 99352, USA

*Corresponding authors (emails: Chunlong.Chen@pnnl.gov (Chen C); lcui@dgut.edu.cn (Cui L))
} 


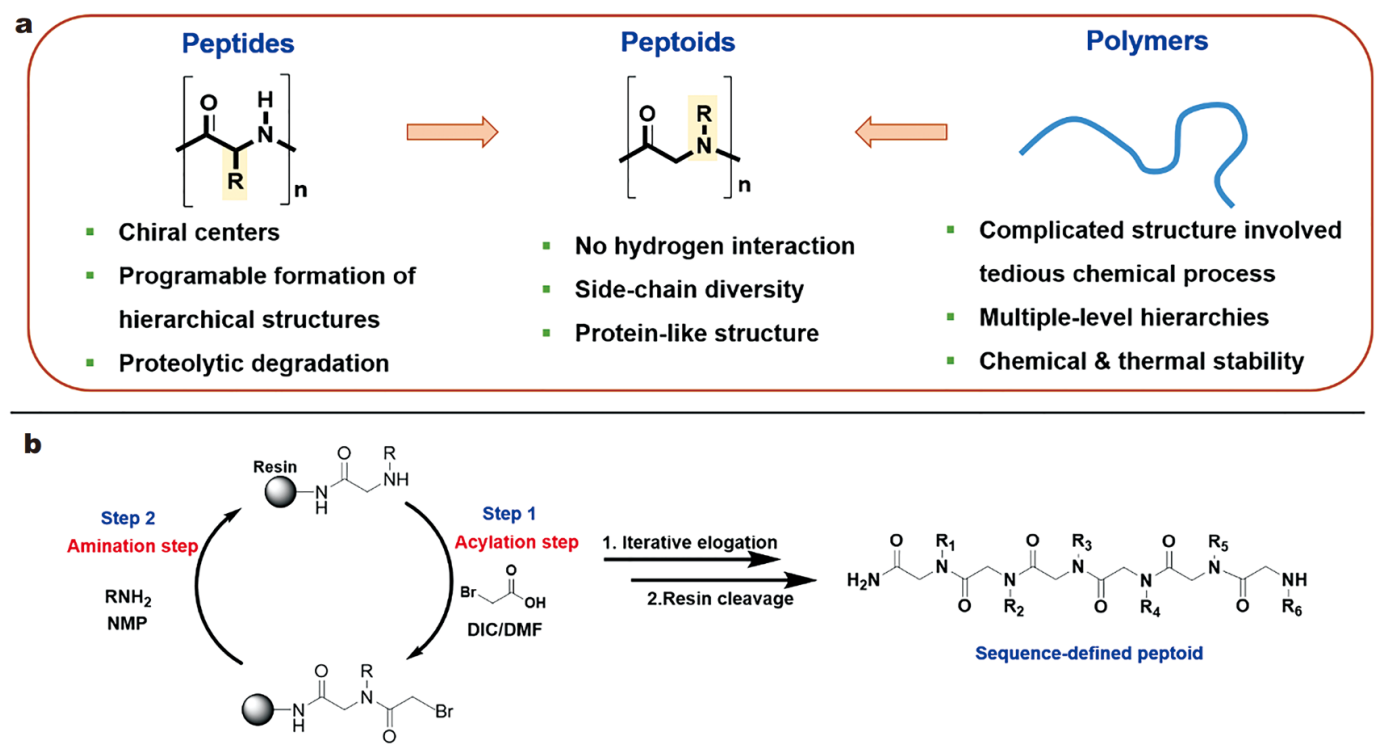

Figure 1 (a) The structures of peptides and peptoids, and peptoids bridge the gap between the natural polymers and synthetic polymers. (b) Solidphase synthesis of sequence-defined peptoids through a repeating two-step submonomer synthesis method.

ods, such as X-ray diffraction (XRD) and dynamic light scattering (DLS), can be used to determine the crystallinity, size and surface charges of peptoid assemblies, they are limited in determining the assembly pathways and dynamics. Atomic force microscopy (AFM) is a beneficial tool with unprecedented spatial resolution and mechanical resolution to investigate the detailed structural parameters in situ under liquid conditions [22,23]. Besides, AFM-based dynamic force spectroscopy (AFMDFS) allows characterizing the thermodynamic and kinetic parameters of the molecular assemblies [24,25]. A combination of in situ AFM imaging with DFS enables direct observation of the assembly process at the nanometer scale and deep understanding of the driving forces that control hierarchical self-assembly [26].

To date, several review articles on the synthesis, properties, and applications of peptoids have been published $[2,27,28]$. Herein, it is not our intention to give a comprehensive overview of the latest research progress in the field of peptoid-based nanomaterials, instead, we will give an overview of some recent achievements in the selfassembly of amphiphilic peptoids into hierarchicallystructured materials, such as nanoribbons $[10,13,16]$, nanomembranes $[14,15]$ and nanotubes $[8,9,12]$, highlighting our fundamental understandings of the peptoid assembly pathway and mechanisms using in situ AFM and AFMDFS techniques. We believe some views and insights from this review will benefit materials scientists for the future design and development of peptoid-based biomimetic na- nomaterials with desired structures and properties.

\section{SURFACE-TEMPLATED PEPTOID SELF- ASSEMBLY}

Inspired by self-assemblies in nature, proteins and peptides have been frequently used as building blocks to assemble functional materials at solid surfaces, such as materials for biocompatible implants and tissue regeneration. However, due to the complexity of protein and peptide folding, the prediction of protein and peptide assembly represents a formidable challenge, and thus the rational design and controlled assembly of proteins or peptides on surfaces remain underdeveloped. Peptoids, due to their lack of backbone hydrogen bonds, enable the tuning of peptoid-peptoid and peptoid-surface interactions exclusively through side-chain chemistry. The exploitation of peptoid side-chain interactions for the $\beta$ sheet-like assembly was first reported by Nam et al. [29] in 2010, where they used charged hydrophilic side chains that form electrostatic interactions and built complementary sequences by alternating them with the hydrophobic side chains. The assembly of these peptoids into nanosheets was made possible through an interfaceassisted process of monolayer compression [29-31]. Zuckermann and colleagues [32,33] later used this design principle to develop self-complementary sequences that assembled into nanosheets. A similar strategy was also used to design 30-mer amphiphilic peptoids that assembled into homochiral superhelices [34]. Inspired by 
these studies, Chen et al. [16,35] recently designed similar peptoid sequences with $\mathrm{N}$-[2-(4-chlorophenyl)ethyl]glycine $\left(\mathrm{N}_{4-\mathrm{Cl}} \mathrm{pe}\right)$ hydrophobic sidechains and used $\mathrm{Ca}^{2+}$ carboxylate interactions to build $\beta$-sheet-like inter-peptoid interactions for mica-templated peptoid self-assembly.

\section{Mica-templated formation of hexagonally patterned networks of nanoribbons}

Design of peptoid and self-assembly into nanoribbons In specific, 12-mer peptoid (Pep-1) with alternating polar $\mathrm{N}$-(2-carboxyethyl)glycines ( $\mathrm{Nce}$ ) and nonpolar $\mathrm{N}_{4-\mathrm{Cl}}$ pe side chains (Fig. 2a) were synthesized and self-assembled into hexagonally patterned networks of nanoribbons on mica surfaces. When this peptoid was dissolved in Tris buffer at $\mathrm{pH}$ 8.0, the Nce groups became fully deprotonated and could coordinate with $\mathrm{Ca}^{2+}$ cations in terms of inter-peptoid and peptoid-mica interactions while the aromatic $\mathrm{N}_{4-\mathrm{cl}}$ pe side chains offered hydrophobic interactions, thus driving the formation of hexagonally patterned nanoribbons on mica surfaces.

The mechanism of mica-templated nanoribbon formation To investigate the detailed assembly pathways and mechanism of this mica-templated peptoid self-assembly system [16,35], in situ AFM imaging and AFM-DFS were a Pep-1
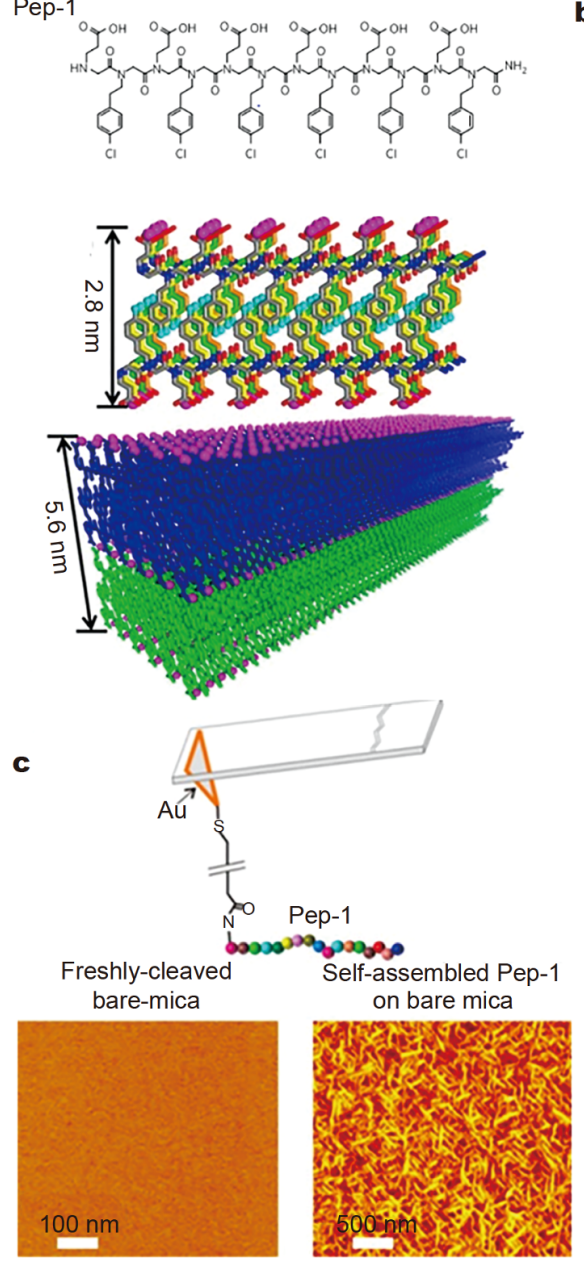

b
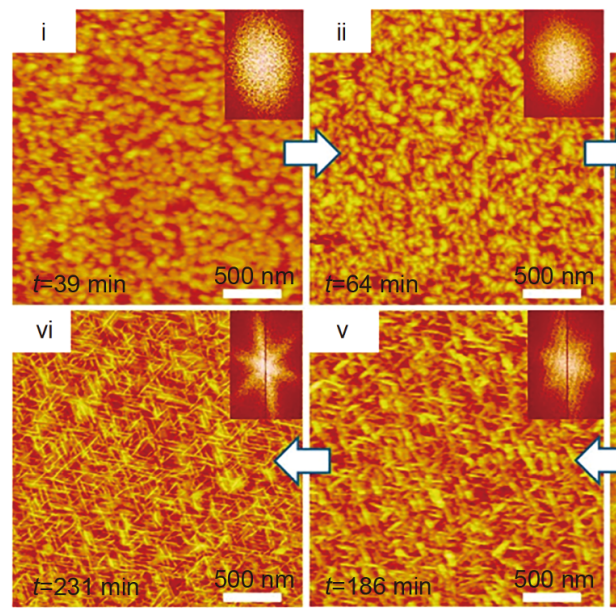

iii
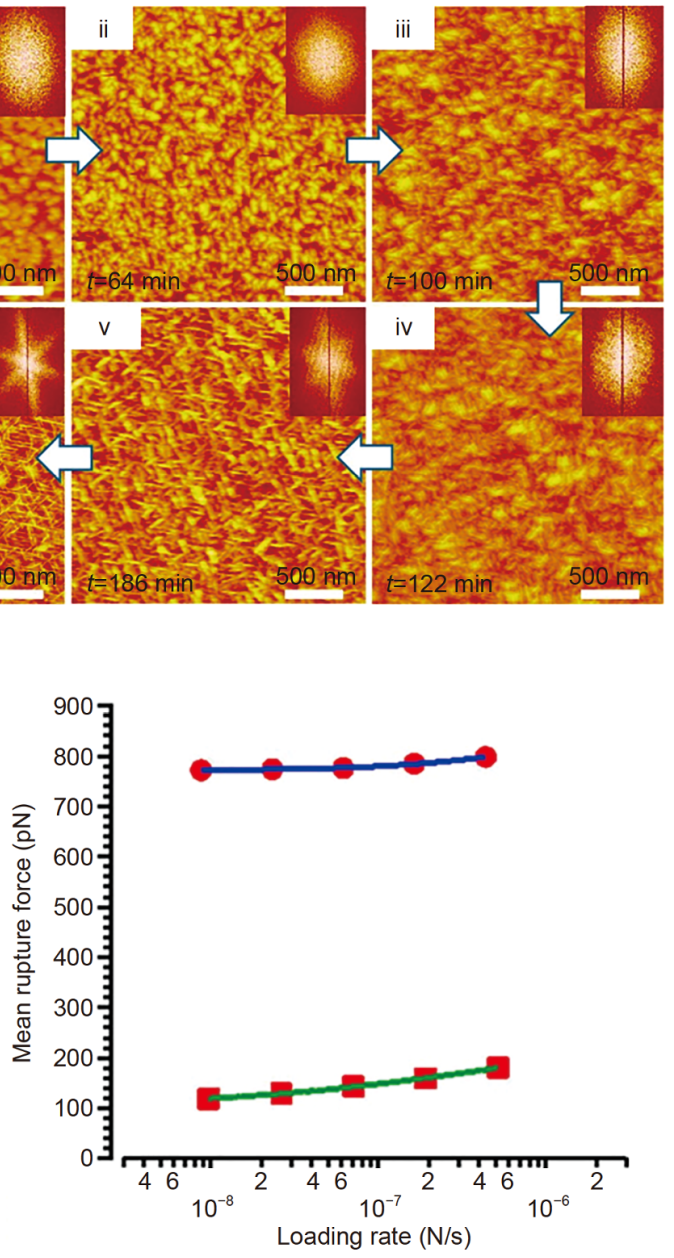

Figure 2 (a) Structure of Pep-1 and a proposed model showing the multilayered peptoid ribbons connected through Ca ${ }^{2+}$-carboxylate coordination bonds and hydrophobic interactions (nitrogen, blue; oxygen, red; chloride, cyan; calcium cation, purple). (b) Time dependent in situ AFM images showing Pep-1-Ca ${ }^{2+}$ complexes assembly pathway on mica surface, in which Pep-1-Ca ${ }^{2+}$ nanoparticles were directly transformed into hexagonally patterned nanoribbons. The inset of each image is a 2D Fourier transform showing the six-fold symmetry. (c) Dynamic force spectra of Pep-1modified AFM tips with bare mica or preassembled Pep-1. Fitting the mean rupture forces between Pep-1 and bare mica (top curve) or between Pep-1 and preassembled Pep-1 on mica (bottom curve) yields the single-molecule binding free energy. Reprinted with permission from Ref. [16], Copyright 2016, American Chemical Society. 
used. Specifically, Pep-1- $\mathrm{CaCl}_{2}$ solution was injected into the AFM fluid cell, and the obtained in situ AFM results showed that the Pep-1-Ca ${ }^{2+}$ solution initially presented as discrete nanoparticles on the mica surface. As time continued, these nanoparticles transformed into oriented, nanorod-like structures (Fig. 2b). The two-dimensional (2D) Fourier transform of the time-dependent in situ AFM images showed that all resulting nanoribbons were packed hexagonally after 186-minute incubation and formed a highly ordered network structure. Besides, only discrete nanoparticles of Pep-1-Ca ${ }^{2+}$ complexes were observed without introducing mica surfaces, indicating that the peptoid-mica interaction is critical for forming this ordered network structure. To better understand the roles of peptoid-mica and peptoid-peptoid interactions, AFM-DFS was used to acquire the force-distance curves and obtain the single-molecule binding free energy of peptoid-mica and peptoid-peptoid. The binding free energy for peptoid-mica $\left[\Delta G_{(\text {pep-mica })}\right]$ was $-17.7 \pm 1.5 k_{\mathrm{B}} T$ and the peptoid-peptoid binding free energy $\left[\Delta G_{(\text {pep-pep })}\right]$ was $-7.8 \pm 0.4 k_{\mathrm{B}} T$ (Fig. $2 \mathrm{c}$ ), showing that peptoid bound to mica surface much stronger than preassembled peptoid nanoribbons. These DFS results further demonstrated that the peptoid-mica interaction was sufficiently strong to drive the transformation of discrete nanoparticles to hexagonally patterned nanoribbons.

Because peptoid assembly is determined by various driving forces (e.g., hydrophobic interactions, cations), a modification of self-assembling peptoids with different heads, or tails can significantly influence peptoid assembly pathways. For example, when a hydrophobic tail was attached at the end of Pep-1 to synthesize Pep-2 (Fig. 3a), in situ AFM was used to directly monitor the whole assembly process of Pep-1 and Pep-2, from the initial state mixing both sequences with the $\mathrm{CaCl}_{2}$ solution to the formation of the patterned nanoribbon networks. In situ AFM results showed that the peptoid assembly pathways are sequence dependent [35], and the attachment of hy-
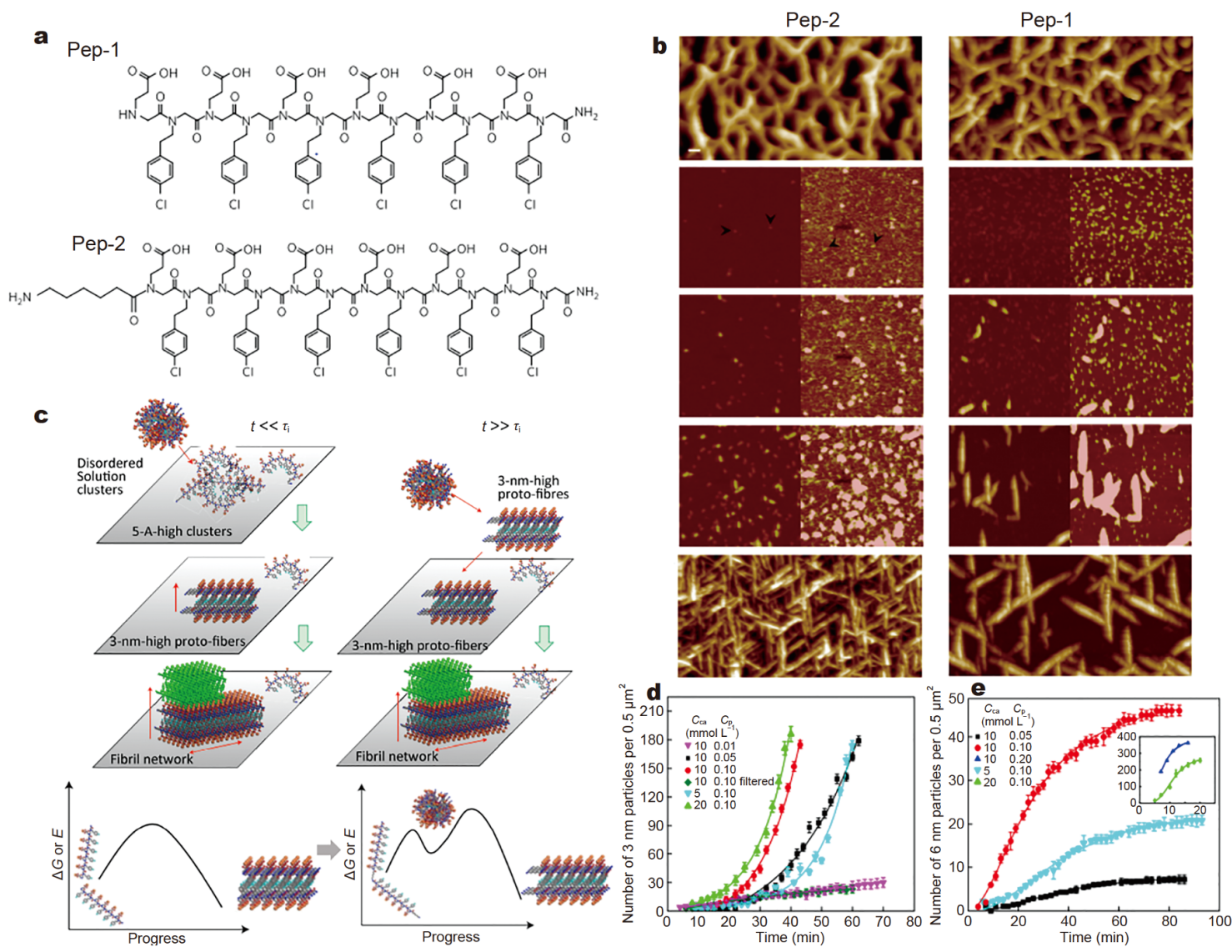

Figure 3 (a) Structures of Pep-1 and Pep-2. (b) In situ AFM imaging of Pep-2 (left) and Pep-1 (right) assembly process to form porous networks. (c) Proposed model for Pep-2 assembly process at early (left) and late (right) stages showing the effect of hydrophobic conjugate on the propensity for peptoid aggregation. (d, e) Kinetics of crystal formation. Nuclei number density versus time for Pep-2 (d) and Pep-1 (e). Reprinted with permission from Ref. [35], Copyright 2017, Nature Publishing Group. 
drophobic tail to the end of Pep-1 resulted in the switching of the assembly pathway from a single-step to a two-step process. Specifically, Pep-1 exhibited a simple single-step assembly pathway, and only 3-nm-high particles were observed (Fig. $3 \mathrm{~b}$ and c). Pep-2 showed an interesting two-step crystallization pathway which began with 5 - $\AA$-high clusters. These clusters were then converted into 3-nm-high nuclei particles before ultimately forming a three-dimensional porous network. The kinetics of peptoid assembly were also determined in different concentrations of peptoids and $\mathrm{Ca}^{2+}$. For Pep-1, the formation rates decreased with time in accordance with a simple Langmuir dependence and were dependent on both peptoid and $\mathrm{Ca}^{2+}$ concentrations (Fig. 3d). Within contrast to Pep-1, at relatively high Pep-2 concentrations, the formation rate of 3-nm-high particles transitioned from a low to high value after a time $\tau$ that decreased with the increase of both peptoid concentration and $\mathrm{Ca}^{2+}$ concentration. However, at low Pep-2 concentrations, no such transition was observed, and the formation rates remained low (Fig. 3e). After the transition, the formation rate increased with Pep-2 concentration but was independent of $\mathrm{Ca}^{2+}$ concentrations. Pep1-Pep1 and Pep1mica binding free energies under different $\mathrm{Ca}^{2+}$ concentrations were determined by AFM-DFS. These AFMDFS results showed the opposite dependence of $\mathrm{Ca}^{2+}$ concentration: an increase of $\mathrm{Ca}^{2+}$ concentration led to a decreased binding of Pep-1 to the surface, providing a reasonable explanation for peptoid crystallization kinetics on $\mathrm{Ca}^{2+}$ concentration. These findings based on in situ AFM and AFM-DFS shed new light on crystallization mechanisms and have implications for the design of selfassembled peptoid materials.

\section{$\mathrm{SiO}_{2}$-templated self-assembly of cylindrical micelles}

Developing synthetic systems for supramolecular self-assembly of hierarchical structures to achieve hierarchy by functionalizing precise atomic locations and incorporating complex sequence information into current synthetic platforms is limited. In a recent work, a significant challenge in the self-assembly of peptoids with hierarchical structures was reported by using one previously reported lipid-like peptoid with a site-specific functionalization.

Design of peptoid and self-assembly of cylindrical micelles Recently, Jin et al. $[14,15,36]$ reported that the lipid-like peptoid (Nce) ${ }_{6}\left(\mathrm{~N}_{4-\mathrm{Cl}} \mathrm{pe}\right)_{6}$ (Pep-3) self-assembled into highly stable and self-repairable membrane-mimetic $2 \mathrm{D}$ nanomaterials. The incorporation of $\beta$-cyclodextrin (CD) headgroup into the hydrophilic domain brought the opportunity to access various self-assembled structures [37]. Specifically, we investigated the Pep-3-CD (Fig. 4a) assembly on a few different substrates (such as quartz with uncharged surface and mica with a negatively charged surface) at $\mathrm{pH}$ values in the range of 2.1 to 7.5 and $\mathrm{Ca}^{2+}$ concentrations in the range of 5 to $50 \mathrm{mmol} \mathrm{L}^{-1}$. Pep-3$\mathrm{CD}$ self-assembled into various hierarchical structures from 1D worm-like or cylindrical micelles to 2D membranes to 3D intertwined ribbons dependent on the substrate and solution conditions [10].

The mechanism of $\mathrm{SiO}_{2}$-templated formation of cylindrical micelles

In situ AFM was used to monitor the pathways and kinetics of Pep-3-CD assembly. The Pep-3-CD assembly on $\mathrm{SiO}_{2}$ surface exhibited a worm-like micelle structure without a particular orientation. This is mainly due to the introduction of $\mathrm{CD}$ group in the peptoid hydrophilic domain, resulting in increased electrostatic repulsion between the hydrophilic blocks. A proposed model shows that Pep-3-CD monomers are orderly packed with the $\mathrm{CD}$ headgroups pointing outward and the hydrophobic $\mathrm{N}_{4-\text {-cl }}$ pe tails organizing in the core (Fig. $4 \mathrm{a}$ ). This model was confirmed by the Pep-3-CD assemblies at different $\mathrm{pH}$ conditions. While Pep-3-CD assembled into cylindrical micelles on quartz surfaces at $\mathrm{pH}$ values between 2.1 and 2.8, Pep-3-CD monomers remained in small particles at higher $\mathrm{pH}$ because the Nce residues were deprotonated and the electrostatic repulsion was increased. At lower $\mathrm{pH}$, the Pep-3-CD solubility significantly decreased, resulting in the formation of large aggregates. We tracked the formation process of Pep-3-CD cylindrical micelles on $\mathrm{SiO}_{2}$, and in situ AFM results showed a two-step selfassembly process, starting with $3-4 \mathrm{~nm}$ spheroidal precursors and then aggregated and transformed into larger worm-like micelles 6-7 nm in size. Finally, these micelles continued to grow until forming the final cylindrical micelles (Fig. 4b and d). A remarkable fact is the Pep-3$\mathrm{CD}$ assembly process on mica is quite different from that on the $\mathrm{SiO}_{2}$ surface. In situ AFM study of Pep-3-CD assembly on mica showed that Pep-3-CD firstly formed highly convoluted worm-like micelles, then the coverage of Pep-3-CD micelles continued to increase and eventually completely covered the surface to form 2D films with a thickness of $\sim 4.5 \mathrm{~nm}$ which is similar to the thickness of $2 \mathrm{D}$ membrane (Fig. $4 \mathrm{c}$ and d). When $\mathrm{Ca}^{2+}$ was introduced, the 3D intertwined ribbon was formed on top of the $2 \mathrm{D}$ film, demonstrating that $\mathrm{Ca}^{2+}$ served as a bridge to facilitate the interactions between $\mathrm{CD}$ head- 

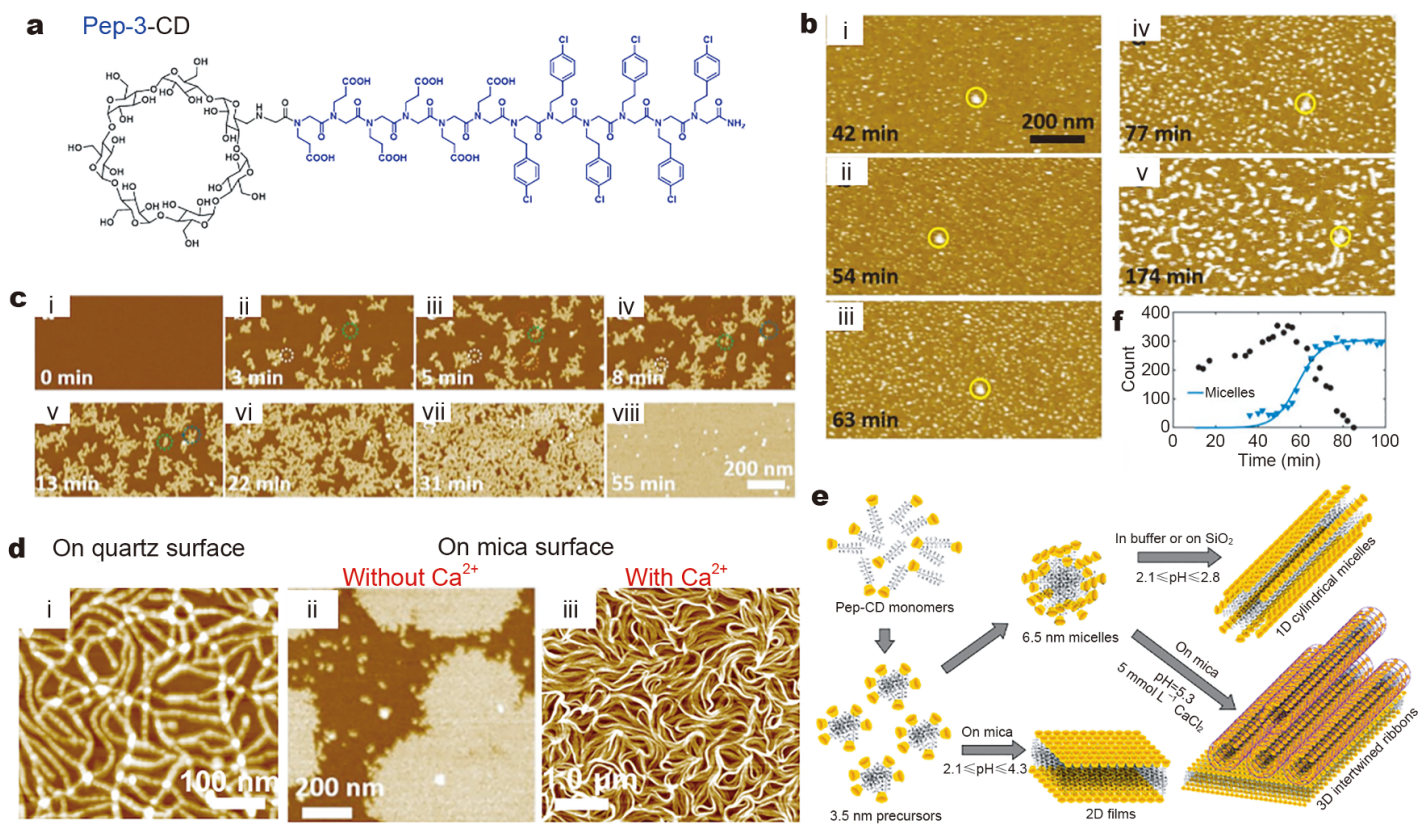

Figure 4 (a) Structures of Pep-3-CD. (b) In situ AFM time series showing two-step assembly of Pep-3-CD cylindrical micelles and (f) kinetics of spheroidal precursors (black) transformed into cylindrical micelles (blue) on quartz. (c) In situ AFM time series images showing Pep-3-CD assembly into 2D films on mica at $\mathrm{pH}$ 2.8. (d) AFM images showing Pep-3-CD self-assembled into 1D cylindrical micelles on quartz surface (i), 2D films on mica surface (ii) and 3D intertwined ribbons with $5 \mathrm{mmol} \mathrm{L}^{-1} \mathrm{CaCl}_{2}$ on mica surface (iii). (e) Illustration of the mechanisms of Pep-3-CD selfassembly into cylindrical micelles, 2D films and 3D intertwined ribbons. Reprinted with permission from Ref. [10], Copyright 2019, Wiley.

groups and carboxylate groups and drive the assembly of Pep-3-CD cylindrical micelles into the final 3D intertwined ribbons (Fig. 4d). In situ AFM results further revealed the assembly kinetics of Pep-3-CD film formation on mica. The rate of coverage is dependent on the peptoid concentration, solution $\mathrm{pH}$ and $\mathrm{Ca}^{2+}$ concentration. Higher peptoid concentration levels increased the rate of coverage, while an increase of solution $\mathrm{pH}$ slowed the kinetics of assembly. The introduction of low $\mathrm{Ca}^{2+}$ concentration facilitated the assembly, but a high concentration of $\mathrm{Ca}^{2+}$ had strong binding to the Pep-CD monomer, which delayed the formation of the worm-like micelle precursors and reduced the rate of coverage. These in-situ AFM results described a picture of Pep-3CD self-assembly into cylindrical micelles, 2D films and 3D intertwined ribbons (Fig. 4e) and provided insight of mechanism and kinetics of peptoid self-assembly into hierarchical structures.

\section{SELF-ASSEMBLY OF PEPTOIDS INTO CRYSTALLINE NANOMATERIALS IN SOLUTION}

\section{Peptoid-based crystalline nanomembrane}

Owing to their unique structures and intrinsic physical and chemical properties, 2D nanomaterials have recently attracted intense interest as promising materials in a broad range of applications in photovoltaics, electronics, chemical sensing, catalysis, water filtration and also in biomedical areas [7,37-39]. Molecular self-assembly offers a unique 'bottom-up' approach to build up welldefined functional 2D nanostructures [36]. Jin et al. $[14,15]$ recently reported the first example of selfassembled highly stable and self-repairing membranemimetic $2 \mathrm{D}$ nanomaterials by designing lipid-like sequence-defined peptoids with aromatic hydrophobic sidechains.

\section{Design of peptoid and self-assembly of nanomembrane}

Specifically, Jin et al. [14] designed amphiphilic lipid-like peptoids (Pep-3) consisting of one block with six polar Nce residues, and another with six nonpolar $\mathrm{N}_{4-\mathrm{cl}} \mathrm{pe}$ residues (Fig. 5a). The self-assembly of Pep-3 was induced by an evaporation-induced crystallization process, and $e x$ situ AFM data showed that gel-like materials formed after the crystallization process, which contained a large amount of 2D nanosheets with straight edges (Fig. 5a) and a thickness comparable to the cell membranes. XRD data showed that these peptoid-based nanomembranes were highly crystalline. These peptoid membranes were highly 
a
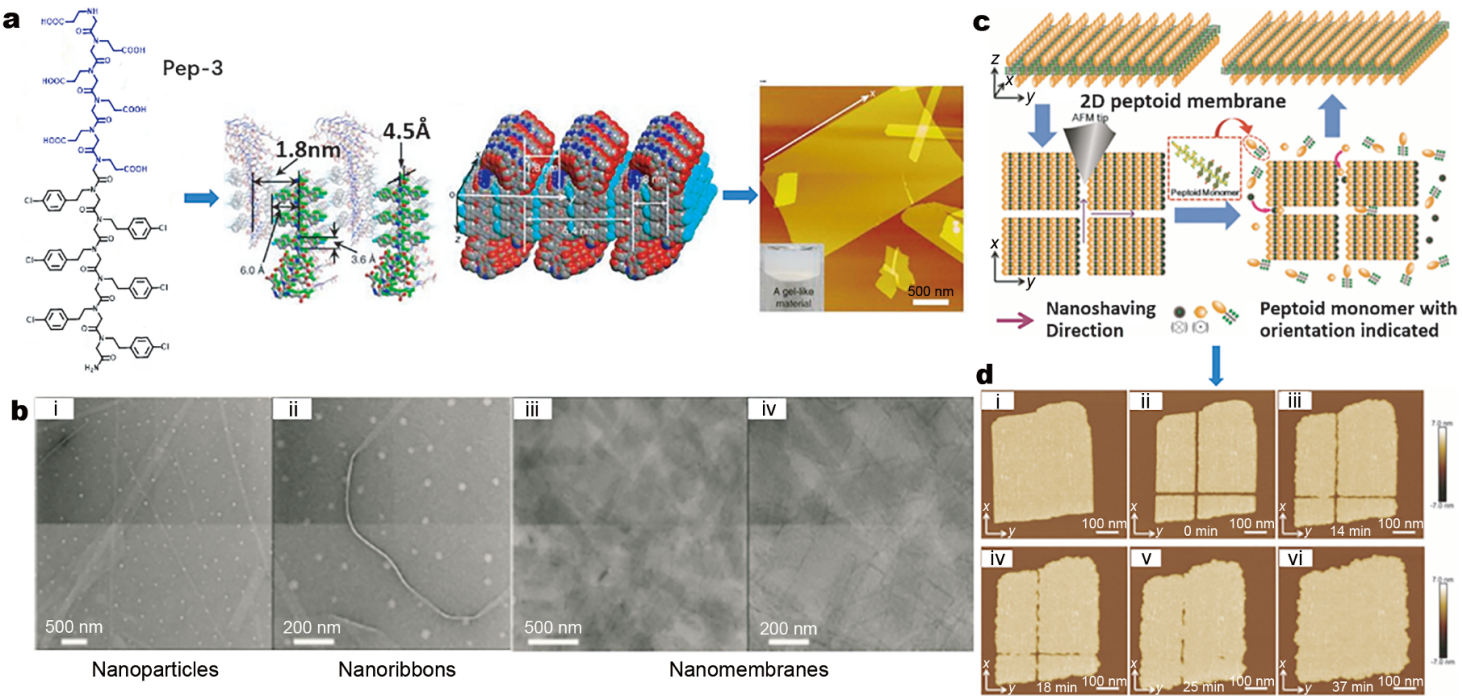

Figure 5 (a) Structure of Pep-3 and the scheme showing its assembly into membrane-mimetic 2D nanomaterials, and an AFM image of Pep-3 nanomembranes with straight edges. (b) TEM images showing the elongated nanoribbon intermediates before the nanomembrane formation. (c) Schematic illustration of the AFM mechanical manipulation and peptoid membrane repair process. (d) In situ AFM images showing the self-repair process of peptoid membranes on mica surface. Reprinted with permission from Ref. [15], Copyright 2016, Wiley.

stable and exhibited a salt-induced thickness change.

\section{Peptoid nanomembrane formation mechanism}

Time-dependent transmission electron microscopy (TEM) and XRD characterizations were utilized to investigate the nanomembrane formation process, these results showed that the amphiphilic peptoids started as amorphous spherical particles in solution. These particles were then transformed into elongated nanoribbons before they were finally converted into highly crystalline nanomembranes (Fig. 5b). Based on AFM, TEM, and XRD results, a model showing the packing of peptoids to form 2D nanomembrane was proposed, in which we reasoned that peptoid membranes were stabilized by the enhanced hydrophobic interactions, such as $\pi-\pi$ interaction as a result of ordered packing of hydrophobic $\mathrm{N}_{4-\mathrm{Cl}}$ pe side chains.

\section{Self-repair of peptoid nanomembrane}

Peptoid nanomembranes exhibit a variety of unique properties similar to cell membranes, such as the ability to self-repair. To demonstrate that, a widely-used AFM lithography-based mechanical scratch method [40] was used to create defects along both $x$ - and $y$-directions of the peptoid nanomembrane (Fig. 5c). After introducing the pre-assembled peptoid solution, in situ AFM was then used to monitor the repairing process in real time. AFM results showed that the defects were completely repaired after $40 \mathrm{~min}$, and the repair rate along the $x$-direction was faster than that along the $y$-direction (Fig. $5 \mathrm{~d}$ ). To better understand such a self-repairing property, three different substrates, atomically flat mica which is hydrophilic and negatively charged, mica modified by aminopropyl silatrane (APS) which is positively charged, and highly ordered pyrolytic graphite which is hydrophobic, were used. In situ AFM-based repairing results showed that the peptoid nanomembranes self-repaired on both mica and APS-mica surfaces, and even without the surface support. The detailed kinetic data generated from in situ AFM studies showed that the self-repair is a two-stage anisotropic process, in which the repair during the first stage is an order of magnitude slower than that in the second stage. Moreover, when a peptoid with the same hydrophobic block and different hydrophilic tails was introduced to the defect-containing nanomembranes, the repair was also observed and the added peptoids formed a new pattern in the nanoscale defect area, further demonstrating the self-repair ability to create nanoscale patterns of functional groups within peptoid-based nanomembranes. In situ AFM results further showed that the peptoid concentration or $\mathrm{pH}$ of the repairing solution has a significant impact on the membrane repairing rates. As indicated by these repairing results, AFM was demonstrated as a suitable tool to create nanoscale patterns for the nanomembranes, and it offered a direct observation of self-repair process and nanoscale patterning of 
functional groups in real time, providing us with new insights into the understanding of membrane self-assembly and self-repair mechanisms.

\section{Peptoid-based crystalline nanotube}

Hollow tubular nanostructures offer a variety of applications in chemistry, biochemistry and materials science as specific molecular reaction vessels [41], screening activities of membrane channels and the nanoscale fluidic transport systems [42]. However, highly stable and programmable organic nanotubes (ONT) are very rare $[43,44]$. Recently, by changing the hydrophobic domains of self-assembling peptoids, we designed and synthesized a new class of highly tunable and dynamic stiff nanotubes formed through a unique rolling-up and closure of the nanosheet mechanism [12].

\section{Design of peptoid and self-assembly of nanotubes}

When the hydrophobic block $\left(\mathrm{N}_{4-\mathrm{Cl}} \mathrm{pe}\right)_{6}$ residues of membrane-forming peptoid were replaced with five to seven residues of $\mathrm{N}$-[(4-bromophenyl)methyl]glycines $(\mathrm{Nbpm})_{6}$ (Pep-4, Fig. 6a), the resulting peptoids selfassembled into single-walled peptoid nanotubes (SWPNTs) under a similar evaporation-induced crystallization process. AFM, TEM and XRD studies showed that the nanotubes were highly crystalline and stable, and they survived the exposure to high temperature $\left(60^{\circ} \mathrm{C}\right)$ and organic solvents $\left(\mathrm{CH}_{3} \mathrm{CN}\right.$ and $\left.\mathrm{EtOH}\right)$.

\section{Peptoid nanotube formation mechanism}

In order to obtain a better view of the intermediate state of PNT formation, the crystallization rate was slowed down by reducing the initial peptoid concentration from 5.0 to $0.5 \mathrm{mmol} \mathrm{L}^{-1}$. Time-dependent TEM results showed an assembly process beginning with homogeneous nanoparticles with a diameter of $40 \mathrm{~nm}$. As the solvent evaporated, these particles crystallized to form nanoribbons. After $24 \mathrm{~h}$, the nanoribbons rolled up from both edges and closed up to completely form nanotubes in $72 \mathrm{~h}$ (Fig. 6b). Based on that, we reasoned that the PNTs were formed through a rolling-up, folding and closure of nanoribbon mechanism through hydrophobic interactions between the aromatic residues. We further demonstrated the tuning of PNT diameter and wall thickness by changing the number of Nbpm groups. Although ex-situ TEM offers a clear picture of the PNT formation process, the detailed evolution of these intermediates remains unclear. We are currently working on using in situ AFM to investigate the self-assembly pathways and mechanisms of the PNT formation.

\section{Dynamic feature of peptoid-based nanotubes}

Because liquid-cell AFM enables the direct monitoring of the changes and dynamics of soft matters in a liquid environment, and tube-forming peptoids exhibit $\mathrm{pH}$ dependent deprotonation and protonation, liquid-cell AFM was also used to monitor the dynamic behaviors of PNTs in response to solution $\mathrm{pH}$ [12]. Specifically, when the solution $\mathrm{pH}$ changed from 8.0 to 3.6 , and then to 8.0 again, AFM results showed that the PNT height decreased from 24.3 to $13.1 \mathrm{~nm}$ as solution $\mathrm{pH}$ decreased, and then returned to the original height of $24.3 \mathrm{~nm}$ when the so-
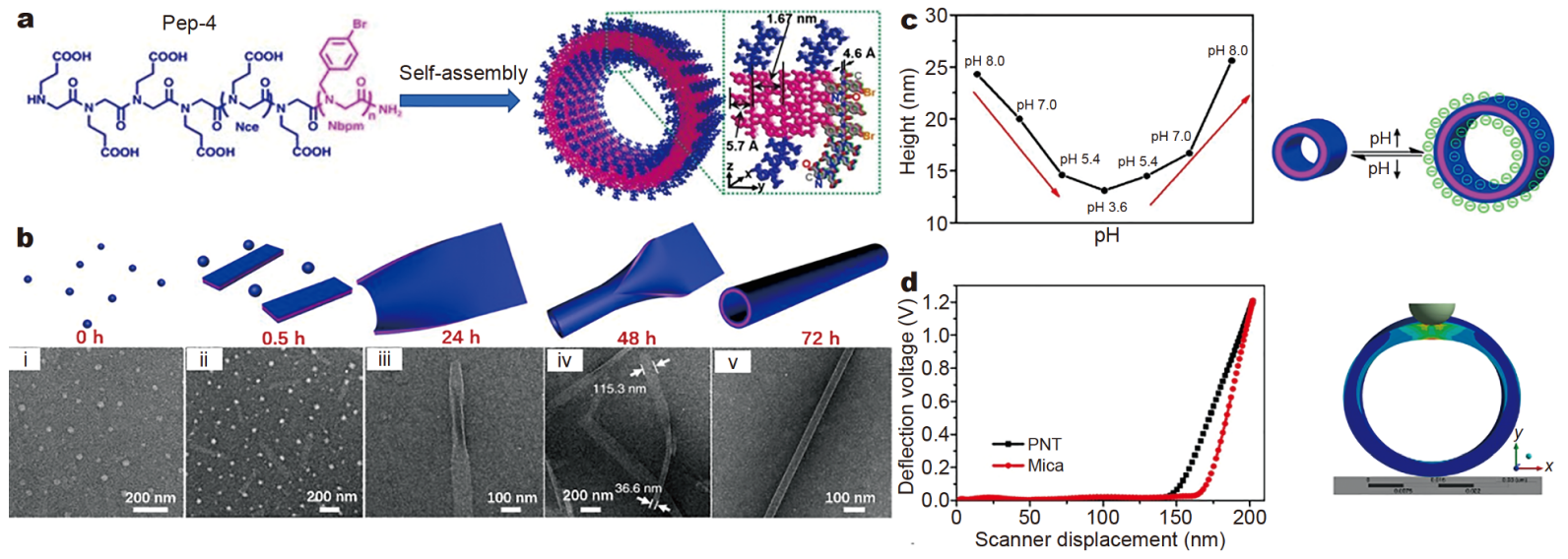

Figure 6 (a) Structure of Pep-4 and a proposed model showing the assembly of Pep-4 into SW-PNTs. (b) Time-dependent TEM images show the assembly process of Pep-PNTs. The Pep-4 assembled into a mixture of nanospheres, then converted into nanosheets with partially rolled up edges, and these sheets further fold and closure to form nanotubes after $72 \mathrm{~h}$. (c) Liquid cell AFM data showing the pH-triggered PNT height changes. (d) AFM-based measurement of the PNT mechanical property, in which the PNTs underwent an intense deformation after an indentation induced by the AFM tip. Reprinted with permission from Ref. [12], Copyright 2018, Nature Publishing Group. 
lution $\mathrm{pH}$ was increased back to 8.0 (Fig. 6c). Such pHresponsive dynamic behavior was fully reversible. When the solution $\mathrm{pH}$ was above 3.3 , the carboxyl group started to deprotonate. Therefore, the electrostatic repulsion was believed to play a key role in the $\mathrm{pH}$-dependent dynamic structural changes of the PNTs.

\section{AFM-based measurement of PNT mechanic property}

The outstanding mechanical property of nanotubes is crucial for their applications. AFM-based nanoindentation is an effective tool to measure the mechanical parameter of nanomaterials. Through the analysis of tip indentation into the sample $\delta$ and the cantilever deflection $\Delta d$, which can be converted to force $F(F=k \times \Delta d, k$ is the spring constant of AFM cantilever), the mechanical properties of the nanomaterial sample (such as Young's modulus) then can be obtained by force-distance curves. Peak force quantitative nanomechanics (PFQNM) is a new AFM technique for measuring Young's modulus of materials [45] at each contact point in real-time with high spatial resolution and surface sensitivity [46]. This technique was recently used to directly probe the PNT's mechanical property in liquid conditions (Fig. 6d). When the applied force increased to $37 \mathrm{nN}$, no damages of the tubular structure were observed for the PNTs assembled from peptoids with six and seven Nbpm groups, and these PNTs returned to their original shapes once the applied forces were removed. In contrast, PNTs assembled from peptoids with five Nbpm groups collapsed when the applied force was over $30 \mathrm{nN}$. These AFMbased nanoindentation results showed that PNTs exhibited Young's modulus in the range of 13 to $17 \mathrm{GPa}$, which is comparable to the stiffest of peptide nanotubes in previous reports [47].

\section{APPLICATIONS OF NANOMATERIALS BASED ON PEPTOID ASSEMBLY}

Self-assembly plays a crucial role in constructing various nanostructures that have potential applications in nanoscience and nanotechnology [48-51]. Self-assembling peptoids provide various building blocks for the fabrication of functional materials with well-ordered nanostructures. Different nanostructures were designed for specific applications. For example, hexagonally patterned nanoribbons offer unique advantages for biomimetic coating [16], nanomembranes could be used for tissue engineering [14] and nanotubes are suitable for biosensor fabrication [12].

\section{As templates to control the formation and assembly of catalytic materials}

Metal nanoparticles have high catalytic activities due to their large surface-to-volume ratio. One major disadvantage is that the metal nanoparticles readily aggregate and thus lose their catalytic activity. Selfassembled peptoid structures were recently used as scaffolds to control the formation of catalytic active Pd nanomaterials [52] (Fig. 7a). When Pep-1 was conjugated with one 6-aminohexanoyl (Aha) at the N-terminus to synthesize $\mathrm{Pep}_{\mathrm{pd}-2}$, this peptoid formed crystalline $2 \mathrm{D}$ nanomembranes at $\mathrm{pH} 2.1$ and less crystalline nanofibers at $\mathrm{pH} 5.6$ [52]. To use these nanomembranes and nanofibers as scaffolds for controlling the formation of $\mathrm{Pd}$ nanomaterials, the stock solution of peptoid nanomem-

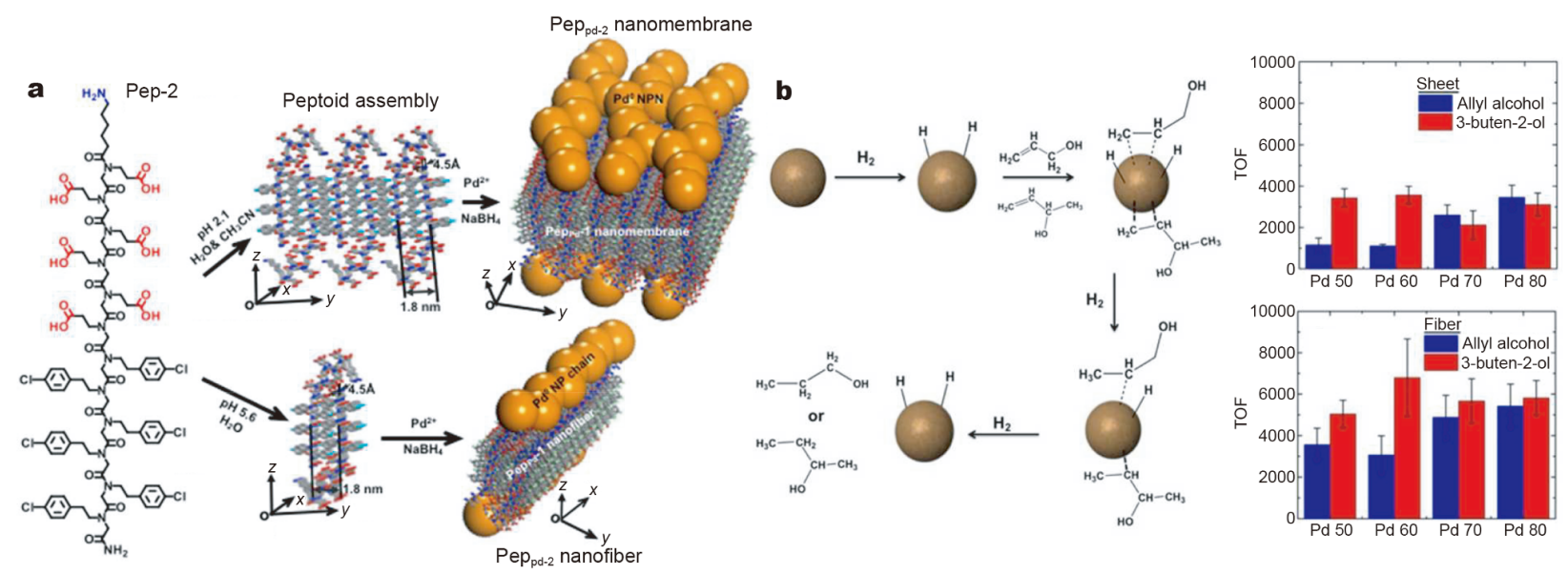

Figure 7 (a) The scheme showing the synthesis of $\operatorname{Pd}^{0}$ nanomaterials templated by peptoid assemblies with two different morphologies. (b) Catalytic analysis of the resulting Pd nanomaterials showing that fiber-templated $\mathrm{Pd}^{0}$ nanomaterials exhibited significant enhancement for olefin hydrogenation over membrane-templated $\mathrm{Pd}^{0}$ materials. Reprinted with permission from Ref. [52], Copyright 2018, Royal Society of Chemistry. 
branes or nanofibers was incubated with potassium tetrachloropalladate (II) $\left(\mathrm{K}_{2} \mathrm{PdCl}_{4}\right)$ which was reduced by sodium borohydride $\left(\mathrm{NaBH}_{4}\right)$ to form the Pd nanomaterials. The catalytic activities of the prepared $\mathrm{Pd}$ nanomaterials were evaluated for the olefin hydrogenation reaction. These catalysis results showed that $\mathrm{Pd}$ nanomaterials templated by $\mathrm{Pep}_{\mathrm{pd}-2}$ nanofibers exhibited higher catalytic activity than the membrane-templated counterparts (Fig. 7b), demonstrating that the morphology of peptoid assembly can significantly influence the properties of templated catalytic nanomaterials.

\section{As cargoes for efficient cytosolic delivery}

Efficient delivery of macromolecule drugs (e.g., nucleic acids, peptides, and proteins) is essential for many genome therapeutic applications. Although non-viral strate- gies including lipid- [53], nanoparticle- [54] and peptidebased methods [55] have shown the improved cellular uptake of therapeutic macromolecule drugs, these approaches are usually problematic, such as high cytotoxicity and low endosomal release efficiency [56]. To address these challenges, we recently designed and synthesized crystalline nanoflower-like nanoparticles assembled from fluorinated peptoids (Pep-5) [17] (Fig. 8a). The cytosolic delivery efficiency of these fluorinated peptoid crystals (FPC) was investigated upon their cellular uptake, toxicity, and cargo release pathway. The cytosolic delivery results showed that these FPCs enabled a nearly $80 \%$ of cytosol delivery rate (Fig. 8b), and a rapid escape from early endosomes without causing pores (Fig. 8d) and minimal cytotoxicity (Fig. 8c). Furthermore, we demonstrated the application of these FPCs as efficient nano-
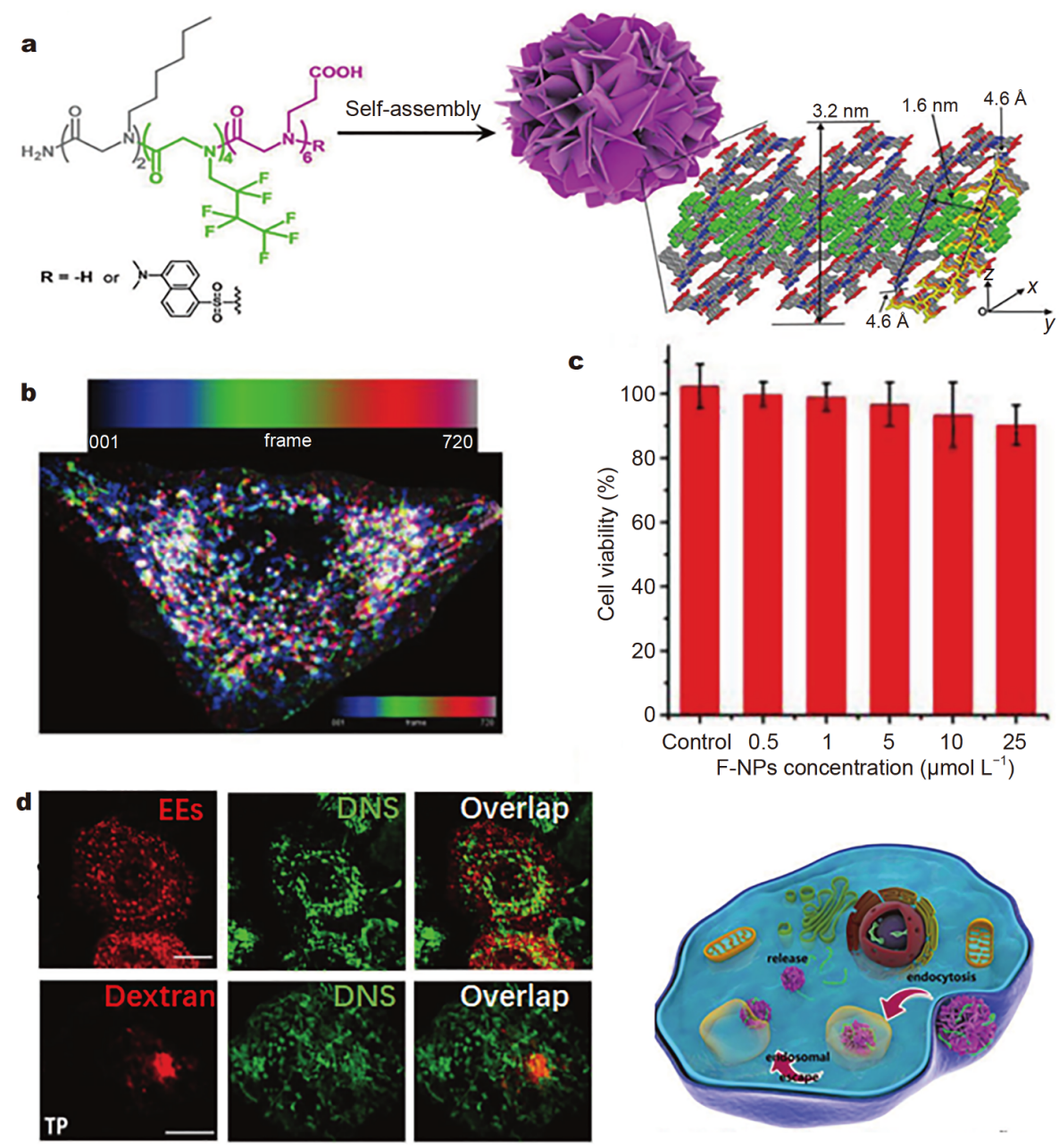

Figure 8 (a) Structure of Pep-5 and the scheme showing self-assembly of fluorinated peptoids into crystalline nanoflowers. (b) Cell incubated with FPC $\left(100 \mathrm{nmol} \mathrm{L}^{-1}\right)$ for $1 \mathrm{~h}$. (c) Cell viability of $\mathrm{H} 1299$ cells after incubating with different concentrations of FPC. (d) Characterization of the localization of the FPCs in the cell. Reprinted with permission from Ref. [17], Copyright 2018, Wiley. 
carriers to deliver model macromolecule drugs, such as ssDNA and mRNA. These FPCs led to a successful intracellular gene expression with a transfection rate as high as around $71 \%$ (Fig. 8 d, right image).

\section{As functional nanomaterials for other applications}

To demonstrate the high tunability of peptoid-based nanomaterials, a variety of functional groups were attached at the N-terminus of either membrane- or tube-forming peptoids, AFM results showed these modifications, including conjugation of self-assembling peptoids with $\mathrm{N}$ [(1-pyrenemethyl)]glycines (Npyr) or CD groups, did not disturb the formation of intact nanomembranes (Fig. 9a, left image) [14] and nanotubes (Fig. 9a, right image) [12]. The high tunability of these peptoid-based nanomaterials enabled various applications. For example, When ArgGly-Asp (RGD) peptides were introduced as functional groups at the N-terminus of tube-forming peptoids, the self-assembled RGD-containing PNTs exhibited an enhanced cellular adhesion and an increased cellular uptake (Fig. 9b). The second example is to precisely engineer PNTs for water decontamination [12]. Due to the large surface area and high porosity of the peptoid nanotubes, functionalized PNTs assembled from CD-conjugated peptoids were able to remove azo-containing dye contaminants from contaminated water, as shown by UV-Vis analysis, demonstrating the potential use of peptoidbased nanomaterials for water purification (Fig. 9c) [12]. Based on the hierarchical self-assembly of Pep-3-CD, the hydrophobic core of these peptoid nanofibers was used as a "host" to accommodate donor molecules (hydrophobic NBD) and the CD cages on the surface of Pep-3-CD cylindrical micelles were used to capture acceptor molecules (Rhodamine B). The synthesized Förster resonance energy transfer (FRET)-active peptoid nanomaterials exhibited a high energy transfer efficiency in aqueous solution (Fig. 9d) [10]. Because functional groups can be precisely incorporated as peptoid side chains, peptoidbased self-assemblies offer a new platform for developing hierarchically-structured biomimetic nanomaterials for applications. Because functional groups can be precisely incorporated as peptoid side chains, peptoid self-assembly offers a new platform for developing hierarchically structured functional nanomaterials. Therefore, besides those applications mentioned above, we expect a wide range of applications for this type of nanomaterials will be reported in the near future.

\section{SUMMARY AND OUTLOOK}

In this review, we have summarized a few recent examples of sequence-defined peptoids that self-assembled into hierarchically-structured nanomaterials, including self-repairable nanomembranes that mimic cell membranes, highly tunable and dynamic single-walled stiff nanotubes formed through a unique "rolling-up and closure of nanosheet" mechanism, and networks of hexagonally patterned nanoribbons templated by mica surfaces. During these hierarchical self-assembly processes,
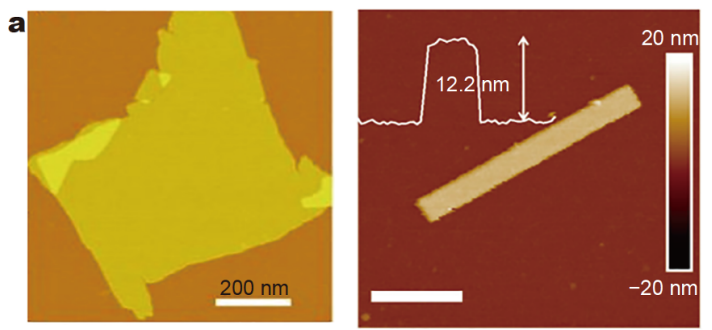

d

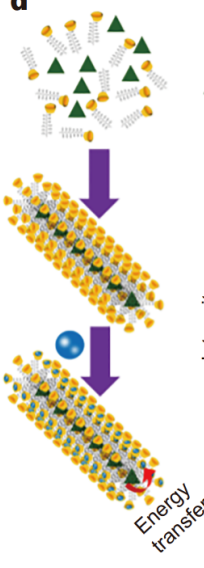

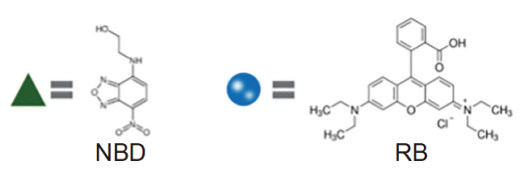

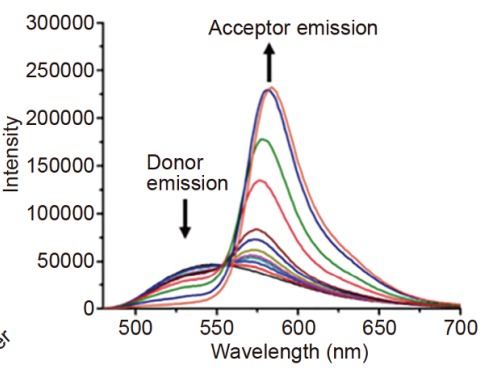

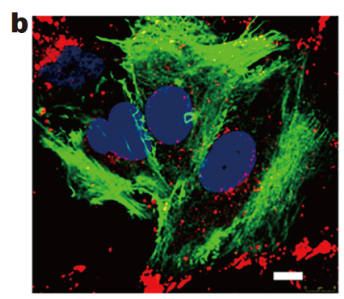

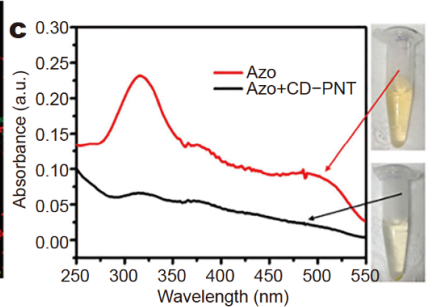

Figure 9 (a) Incorporating functional groups into self-assembling peptoids did not inhibit the formation of nanomembranes (left) and nanotubes (right). (b) Fluorescence image showing that RGD-containing peptoid nanotubes (PNTs) exhibit a high cellular uptake. (c) CD-containing PNTs were used as adsorbents for the removal of azo-dyes from water. (d) The FRET system based on Pep-3-CD cylindrical micelles, NBD donors and RB acceptors. Fluorescence emission results show a high FRET efficiency of this system in aqueous solution. Reprinted with permission from Refs [12,14], Copyright 2016 and 2018, Nature Publishing Group, and Ref. [10], Copyright 2019, Wiley. 
nanoscale characterizations, such as in situ AFM and AFM-DFS, offered critical information for investigating the peptoid assembly pathways and mechanisms, and the properties (e.g., membrane self-repair and mechanical property of PNTs) of the self-assembled nanomaterials. These findings highlight the importance of nanoscale characterizations for understanding peptoid assembly mechanisms and for achieving predictive materials synthesis through controlled peptoid self-assembly. However, there are still many critical issues that need to be addressed for achieving predictable peptoid selfassembly. For example, despite many recent studies showing that peptoid self-assembly has mainly relied on hydrophobic interactions, we still do not fully understand the influence of the polar domain of diblock-like peptoids during their self-assembly. While in situ AFM and AFMDFS offer important insights by monitoring the peptoid assembly pathways and by illustrating the driving forces that determine the peptoid self-assembly, achieving the assembly of peptoid-based nanomaterials with predictable morphologies still faces significant challenges. On the other hand, while the applications of peptoid-based nanomaterials still remain at the infancy stage, the unique features of these nanomaterials, such as highly programmable composition and biocompatibility, open up a wide range of potential applications in sensing, catalysis, tissue regeneration, antimicrobial, and drug delivery.

\section{Received 30 January 2020; accepted 10 March 2020;}

published online 22 April 2020

1 Sun J, Zuckermann RN. Peptoid polymers: A highly designable bioinspired material. ACS Nano, 2013, 7: 4715-4732

2 Knight AS, Zhou EY, Francis MB, et al. Sequence programmable peptoid polymers for diverse materials applications. Adv Mater, 2015, 27: 5665-5691

3 Liu R, Hudalla GA. Using self-assembling peptides to integrate biomolecules into functional supramolecular biomaterials. Molecules, 2019, 24: 1450

4 Lin Y, Mao C. Bio-inspired supramolecular self-assembly towards soft nanomaterials. Front Mater Sci, 2011, 5: 247-265

5 Lutz JF, Ouchi M, Liu DR, et al. Sequence-controlled polymers. Science, 2013, 341: 1238149

6 Chang B, Li X, Sun T. Self-assembled chiral materials from achiral components or racemates. Eur Polym J, 2019, 118: 365-381

7 Robertson EJ, Battigelli A, Proulx C, et al. Design, synthesis, assembly, and engineering of peptoid nanosheets. Acc Chem Res, 2016, 49: 379-389

8 Luo Y, Song Y, Wang M, et al. Bioinspired peptoid nanotubes for targeted tumor cell imaging and chemo-photodynamic therapy. Small, 2019, 15: 1902485

9 Jin H, Jian T, Ding YH, et al. Solid-phase synthesis of three-armed star-shaped peptoids and their hierarchical self-assembly. Biopolymers, 2019, 110: e23258
10 Jiao F, Wu X, Jian T, et al. Hierarchical assembly of peptoid-based cylindrical micelles exhibiting efficient resonance energy transfer in aqueous solution. Angew Chem Int Ed, 2019, 58: 12223-12230

11 Yan F, Liu L, Walsh TR, et al. Controlled synthesis of highlybranched plasmonic gold nanoparticles through peptoid engineering. Nat Commun, 2018, 9: 2327

12 Jin $\mathrm{H}$, Ding YH, Wang M, et al. Designable and dynamic singlewalled stiff nanotubes assembled from sequence-defined peptoids. Nat Commun, 2018, 9: 270

13 Murray DJ, Kim JH, Grzincic EM, et al. Uniform, large-area, highly ordered peptoid monolayer and bilayer films for sensing applications. Langmuir, 2019, 35: 13671-13680

14 Jin H, Jiao F, Daily MD, et al. Highly stable and self-repairing membrane-mimetic 2D nanomaterials assembled from lipid-like peptoids. Nat Commun, 2016, 7: 12252

15 Jiao F, Chen Y, Jin $\mathrm{H}$, et al. Self-repair and patterning of $2 \mathrm{D}$ membrane-like peptoid materials. Adv Funct Mater, 2016, 26: 8960-8967

16 Chen CL, Zuckermann RN, DeYoreo JJ. Surface-directed assembly of sequence-defined synthetic polymers into networks of hexagonally patterned nanoribbons with controlled functionalities. ACS Nano, 2016, 10: 5314-5320

17 Song Y, Wang M, Li S, et al. Efficient cytosolic delivery using crystalline nanoflowers assembled from fluorinated peptoids. Small, 2018, 14: 1803544

18 Zhu L, Zhao Z, Cheng P, et al. Antibody-mimetic peptoid nanosheet for label-free serum-based diagnosis of Alzheimer's disease. Adv Mater, 2017, 29: 1700057

19 Zuckermann RN, Kerr JM, Kent SBH, et al. Efficient method for the preparation of peptoids [oligo(N-substituted glycines)] by submonomer solid-phase synthesis. J Am Chem Soc, 1992, 114: 10646-10647

20 Horn T, Lee BC, Dill KA, et al. Incorporation of chemoselective functionalities into peptoids via solid-phase submonomer synthesis. Bioconjugate Chem, 2004, 15: 428-435

21 Fowler SA, Luechapanichkul R, Blackwell HE. Synthesis and characterization of nitroaromatic peptoids: Fine tuning peptoid secondary structure through monomer position and functionality. J Org Chem, 2009, 74: 1440-1449

22 Malkin AJ, Kuznetsov YG, McPherson A. In situ atomic force microscopy studies of surface morphology, growth kinetics, defect structure and dissolution in macromolecular crystallization. J Cryst Growth, 1999, 196: 471-488

23 Engel A. Atomic force microscopy: A powerful tool to observe biomolecules at work. Trends Cell Biol, 1999, 9: 77-80

24 Takeuchi O, Miyakoshi T, Taninaka A, et al. Dynamic-force spectroscopy measurement with precise force control using atomic-force microscopy probe. J Appl Phys, 2006, 100: 074315

25 Liu J, Zhang X, Wang X, et al. Single-molecule force spectroscopy study of the effect of cigarette carcinogens on thrombomodulinthrombin interaction. Sci Bull, 2016, 61: 1187-1194

26 De Yoreo JJ, Chung S, Friddle RW. In situ atomic force microscopy as a tool for investigating interactions and assembly dynamics in biomolecular and biomineral systems. Adv Funct Mater, 2013, 23: 2525-2538

27 Chan BA, Xuan S, Li A, et al. Polypeptoid polymers: Synthesis, characterization, and properties. Biopolymers, 2018, 109: e23070

28 Battigelli A. Design and preparation of organic nanomaterials using self-assembled peptoids. Biopolymers, 2019, 110: e23265

29 Nam KT, Shelby SA, Choi PH, et al. Free-floating ultrathin two- 
dimensional crystals from sequence-specific peptoid polymers. Nat Mater, 2010, 9: 454-460

30 Robertson EJ, Olivier GK, Qian M, et al. Assembly and molecular order of two-dimensional peptoid nanosheets through the oilwater interface. Proc Natl Acad Sci USA, 2014, 111: 13284-13289

31 Sanii B, Kudirka R, Cho A, et al. Shaken, not stirred: Collapsing a peptoid monolayer to produce free-floating, stable nanosheets. J Am Chem Soc, 2011, 133: 20808-20815

32 Olivier GK, Cho A, Sanii B, et al. Antibody-mimetic peptoid nanosheets for molecular recognition. ACS Nano, 2013, 7: 9276-9286

33 Kudirka R, Tran H, Sanii B, et al. Folding of a single-chain, information-rich polypeptoid sequence into a highly ordered nanosheet. Biopolymers, 2011, 96: 586-595

34 Murnen HK, Rosales AM, Jaworski JN, et al. Hierarchical selfassembly of a biomimetic diblock copolypeptoid into homochiral superhelices. J Am Chem Soc, 2010, 132: 16112-16119

35 Ma X, Zhang S, Jiao F, et al. Tuning crystallization pathways through sequence engineering of biomimetic polymers. Nat Mater, 2017, 16: 767-774

$36 \mathrm{Mu}$ P, Zhou G, Chen CL. 2D nanomaterials assembled from sequence-defined molecules. Nano-Struct Nano-Objects, 2018, 15: 153-166

37 Jariwala D, Sangwan VK, Lauhon LJ, et al. Carbon nanomaterials for electronics, optoelectronics, photovoltaics, and sensing. Chem Soc Rev, 2013, 42: 2824-2860

38 Yang G, Zhu C, Du D, et al. Graphene-like two-dimensional layered nanomaterials: Applications in biosensors and nanomedicine. Nanoscale, 2015, 7: 14217-14231

39 Velický M, Toth PS. From two-dimensional materials to their heterostructures: An electrochemist's perspective. Appl Mater Today, 2017, 8: 68-103

40 Choi CH, Lee DJ, Sung JH, et al. A study of AFM-based scratch process on polycarbonate surface and grating application. Appl Surf Sci, 2010, 256: 7668-7671

41 Balasubramanian R, Prayakarao S, Han S, et al. Tunable gold nanostructures with nanocapsules as template reaction vessels. RSC Adv, 2012, 2: 11668-11671

$42 \mathrm{Xu} \mathrm{WL}$, Smith MD, Krause JA, et al. Single crystal to single crystal polymerization of a self-assembled diacetylene macrocycle affords columnar polydiacetylenes. Cryst Growth Des, 2014, 14: 993-1002

43 Hsieh WH, Liaw J. Applications of cyclic peptide nanotubes (cPNTs). J Food Drug Anal, 2019, 27: 32-47

44 Zhao X, Pan F, Xu H, et al. Molecular self-assembly and applications of designer peptide amphiphiles. Chem Soc Rev, 2010, 39: 3480-3498

45 Young TJ, Monclus MA, Burnett TL, et al. The use of the PeakForce $^{\mathrm{TM}}$ quantitative nanomechanical mapping AFM-based method for high-resolution Young's modulus measurement of polymers. Meas Sci Technol, 2011, 22: 125703
46 Morales-Rivas L, González-Orive A, Garcia-Mateo C, et al. Nanomechanical characterization of nanostructured bainitic steel: Peak force microscopy and nanoindentation with AFM. Sci Rep, 2015, 5: 17164

47 Adler-Abramovich L, Gazit E. The physical properties of supramolecular peptide assemblies: From building block association to technological applications. Chem Soc Rev, 2014, 43: 6881-6893

$48 \mathrm{Yu} \mathrm{SH}$, Chen SF. Recent advances in polymer directed crystal growth and mediated self-assembly of nanoparticles. CNANO, 2006, 2: 81-92

49 Lazzari M, Rodríguez-Abreu C, Rivas J, et al. Self-assembly: A minimalist route to the fabrication of nanomaterials. J Nanosci Nanotech, 2006, 6: 892-905

50 An Y, Ren Y, Bick M, et al. Highly fluorescent copper nanoclusters for sensing and bioimaging. Biosens Bioelectron, 2020, 154: 112078

51 Tang J, Qin N, Chong Y, et al. Nanowire arrays restore vision in blind mice. Nat Commun, 2018, 9: 786

52 Merrill NA, Yan F, Jin H, et al. Tunable assembly of biomimetic peptoids as templates to control nanostructure catalytic activity. Nanoscale, 2018, 10: 12445-12452

53 Aparicio-Blanco J, Sebastián V, Rodríguez-Amaro M, et al. Sizetailored design of highly monodisperse lipid nanocapsules for drug delivery. J Biomed Nanotechnol, 2019, 15: 1149-1161

54 Jiang Y, Tang R, Duncan B, et al. Direct cytosolic delivery of siRNA using nanoparticle-stabilized nanocapsules. Angew Chem Int Ed, 2014, 54: 506-510

55 Schröder T, Niemeier N, Afonin S, et al. Peptoidic amino- and guanidinium-carrier systems: Targeted drug delivery into the cell cytosol or the nucleus. J Med Chem, 2008, 51: 376-379

56 Dowdy SF. Overcoming cellular barriers for RNA therapeutics. Nat Biotechnol, 2017, 35: 222-229

Acknowledgements This work was supported by the Startup Research Fund of Dongguan University of Technology (KCYKYQD2017015) and the US Department of Energy, Office of Science, Office of Basic Energy Sciences, as part of the Energy Frontier Research Centers program: CSSAS-The Center for the Science of Synthesis Across Scales (DESC0019288). Pacific Northwest National Laboratory is a multi-program national laboratory operated for the Department of Energy by Battelle under contract number DE-AC05-76RL01830.

Author contributions Liu J and Chen CL proposed the topic and outline of this review article. Liu J and Cai B collected the related information needed in writing the paper; Liu J, Cui L and Chen CL cowrote and modified the manuscript. All authors discussed and commented on the manuscript.

Conflict of interest The authors declare that they have no conflict of interest. 


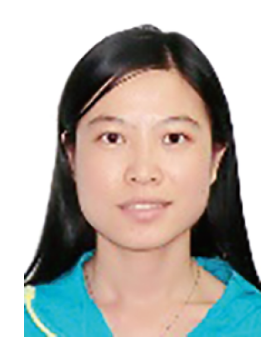

Jianli Liu received her $\mathrm{PhD}$ degree in physical chemistry from the Institutes of Chemistry, Chinese Academy of Sciences in 2016. She is a Postdoc under the supervision of Prof. Lifeng Cui in the School of Materials Science and Engineering, Dongguan University of Technology. Currently, she is working in Prof. Chun-Long Chen's group as a visiting scholar in the Pacific Northwest National Laboratory. Her research interests are the design and assembly of peptoids and the study of their formation mechanisms by in situ AFM.

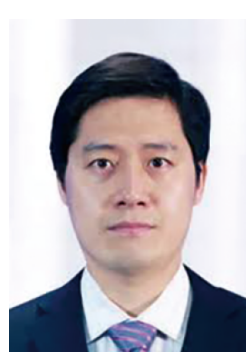

Lifeng Cui finished his undergraduate studies in electrical engineering at Xi'an University of Posts \& Telecommunications in 2001. In 2007, he received his $\mathrm{PhD}$ degree in materials science under the supervision of Prof. Lai-Sheng Wang at Washington State University. After postdoctoral studies in materials science at Stanford University with Professor Yi Cui, he joined Amazon Inc. in 2010 and worked as a R\&D engineer. $\mathrm{He}$ joined Shanghai University for Science \& Technology as a professor in 2013 and moved to his present position as professor of materials science and engineering in 2016. His current research interests include advanced nano-structured materials, photocatalysis, and catalysis for biomass conversion.

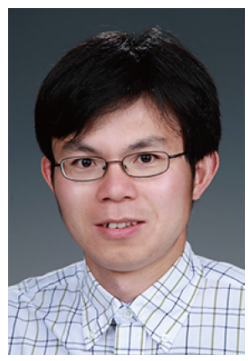

Chun-Long Chen is currently a senior research scientist at the Pacific Northwest National Laboratory (PNNL), and a joint Faculty Fellow in the Department of Chemical Engineering at the University of Washington. His research group is tackling the challenges of developing sequencedefined peptoids that mimic proteins and peptides for assembly of biomimetic functional materials (e.g., artificial membranes) and for controlling inorganic crystal formation (e.g., plasmonic nanomaterials), aiming at design and synthesis of bio-inspired functional materials that rival those found in biology.
基于类肽等级自组装结构仿生纳米材料: 合成, 表 征和应用

刘建丽 ${ }^{1,2}$, 蔡涁 $^{2}$, 崔立峰 ${ }^{1^{*}}$, 陈春龙 ${ }^{2 *}$

摘要 类肽(或 $N$-取代甘氨酸)具有合成效率高、化学稳定性强、 抗酶水解及生物相容性好等优点, 是一类潜在的可自定义序列的 生物仿生聚合物. 通过调节类肽的侧链化学, 可以精确地控制类肽 序列并实现侧链的多样性. 基于以上类肽分子独特的优势, 在过去 的几年里, 研究者设计和合成了大量的双亲性类肽作为基本的结 构单元，通过自下而上的分子自组装作用构建了结构可控和具有 特定功能的仿生纳米材料. 本文从以下三个方面综述了我们在类 肽自组装领域取得的一些成果. 首先, 概述了以云母和硅片为无机 基底辅助类肽自组装. 将原子力显微镜原位成像技术和单分子力 谱技术相结合, 可实时观察类肽分子在表面的组装过程并直接测 定类肽分子与基底间的相互作用，揭示基底表面对类肽自组装的 影响. 其次, 介绍了类肽分子在溶液中自组装成纳米管和具有自修 复功能的纳米薄膜. 最后, 综述了基于类肽自组装纳米材料的应用, 包括以类肽自组装纳米材料为催化模板构建纳米仿生催化剂和作 为细胞内输送物质的载体等. 